\title{
Prescriber adherence to antiemetic guidelines with the new agent trifluridine-tipiracil
}

\author{
Daniel S Childs, MD, ${ }^{a}$ Alison C Jacobson, RN, Jessica L Mitchell, APRN, CNP, ${ }^{\mathrm{b}}$ Joleen M \\ Hubbard, MD, ${ }^{\mathrm{b}}$ Harry H Yoon, MD, ${ }^{\mathrm{b}}$ Heidi Finnes, PharmD, RPh, ${ }^{\mathrm{c}}$ Axel Grothey, MD, \\ and Aminah Jatoi, $\mathrm{MD}^{\mathrm{b}}$
}

Departments of ${ }^{a}$ Medicine and ${ }^{b}$ Oncology, and ${ }^{c}$ Pharmacy Services, Mayo Clinic, Rochester, Minnesota

Background In 2015 alone, the US Food and Drug Administration approved 18 cancer drugs, but to our knowledge, few studies, if any, have examined prescribers' adherence to antiemetic guidelines as new chemotherapy agents become available. This issue is important because poor adherence to antiemetic guidelines has been shown in previous studies to have a negative impact on the control of nausea and vomiting. Here we report on antiemetic practices and outcomes for trifluridine-tipiracil, a drug newly approved in 2015.

Objective To test the hypothesis that patients prescribed a newly available chemotherapy agent, trifluridine-tipiracil, are at risk for chemotherapy-induced nausea and vomiting because of providers' poor adherence to antiemetic guidelines.

Methods All patients who received their first dose of trifluradine-tipiracil for metastatic colon cancer in 2015 were included in this retrospective, single-institution study of pretreated patients. The study time frame was the 2015 calendar year: 9 months before the drug was approved in September 2015, when patients received the medication through a compassionate-use program, and the 3 months immediately after drug approval. First-cycle antiemetic prescribing was examined for adherence to National Comprehensive Cancer Network Guidelines (v1.2015) and categorized as guideline adherent, non-guideline-adherent/aggressive (received more prophylaxis than called for), and non-guideline-adherent/less aggressive (including no antiemetics).

Results Of the 44 patients in this study, $28(64 \%)$ had had nausea and vomiting with previous chemotherapy. With the first cycle of trifluridine-tipiracil, 25 patients $(57 \%$; 95\% confidence interval $[\mathrm{Cl}]: 42 \%, 70 \%)$ were prescribed prophylactic antiemetics in a guideline-adherent manner; $15(34 \% ; 95 \% \mathrm{Cl}: 22 \%, 49 \%)$ in a non-guideline-adherent/aggressive manner; and 4 (9\%; $95 \%$ Cl: $4 \%, 21 \%$ ) in a non-guideline-adherent/less aggressive manner. In guideline-adherent patients, rates of nausea and vomiting were $52 \%$ and $24 \%$, respectively. In non-guideline-adherent/aggressive patients, those rates were $33 \%$ and $27 \%$, respectively. In both the aforementioned groups, a total of 2 patients received interim care for nausea and vomiting. No nausea or vomiting was reported among non-guideline-adherent/less aggressively managed patients.

Limitations Single-institution, retrospective study of a small group of patients

Conclusions Poor adherence to antiemetic guidelines was common. However, because adherence was not consistently associated with better control of nausea and vomiting, clinical judgment should complement guideline adherence when prescribing trifluridine-tipiracil and other newly approved cancer drugs.

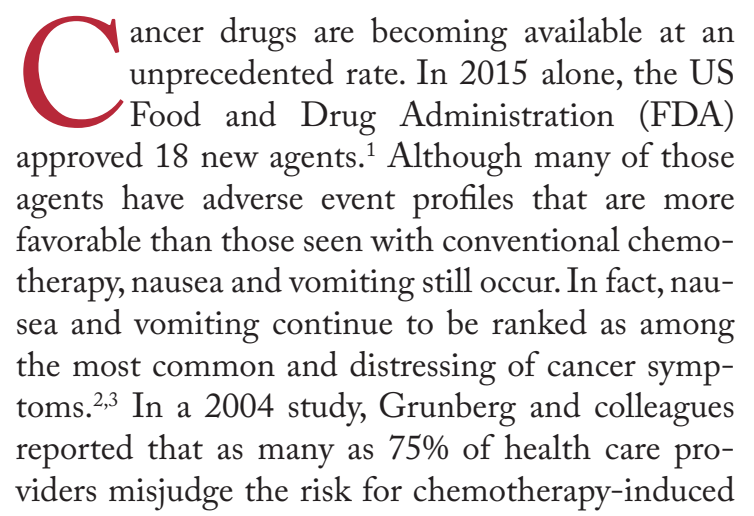

nausea and vomiting (CINV), even when prescribing cancer drugs that have been available for years, ${ }^{4}$ thus amplifying concerns that such risk assessment might be even worse when new cancer agents are prescribed for the first time.

In this study, we hypothesized that patients prescribed a new cancer drug, trifluridine-tipiracil, would be at risk for CINV because of poor guideline adherence on the part of health care providers. The correct matching of antiemetics to chemotherapy is important. Inadequate antiemetic prophylaxis predisposes to nausea and vomiting with dehydration and met-

Accepted for publication November 3, 2016. Correspondence: Aminah Jatoi, MD; Jatoi.aminah@mayo.edu. Disclosures: All of the authors are with the Mayo Clinic, which has received research grants from Taiho Oncology, the maker of trifluridine-tipiracil. JCSO 2017;15(3):e142-e146. (2017 Frontline Medical Communications. doi: https://doi. org/10.12788/jicso.0314 
abolic and electrolyte derangements - complications that can occur in up to one-third of patients who receive moderately or highly emetogenic chemotherapy and who have been reported to achieve poor symptom control. ${ }^{4}$ Overprophylaxis also has drawbacks. For example, antiemetics are expensive and, at times, they can induce their own adverse events, such as lethargy, dyskinesia, constipation, headaches, hiccups, fatigue, and even cardiac arrhythmias. ${ }^{5}$ The best approach is to appropriately match the antiemetic to the chemotherapy. Indeed, adherence to evidence-based guidelines has yielded success in symptom control, but the guidelines work on the assumption that the emetogenic potential of new chemotherapy agents has been accurately determined and then disseminated to and acted upon by health care providers. ${ }^{6,7}$ To our knowledge, no previous studies have tested that assumption, as we do in the present study.

Trifluridine-tipiracil was selected as the focus of this project and as illustrative of other newly approved chemotherapy agents for two reasons. First, it became available for routine prescribing in pretreated patients with metastatic colorectal cancer in the United States in September 2015. That timing allowed us to analyze much of the early prescribing period, both during the 9 months before approval, when the drug was available on a compassionate-use basis at our institution, and the 3 months after approval. Second, trifluridine-tipiracil has classifiably low emetogenic potential, and mismatching of antiemetics tends to occur more often with low emetogenic chemotherapy. ${ }^{9}$ Trifluridinetipiracil and placebo patients manifest rates of nausea at $48 \%$ and $24 \%$, respectively, and rates of vomiting at $28 \%$ and $14 \%$, respectively. ${ }^{8}$

Hence, the goal of this study was to explore whether a guideline-based prophylactic antiemetic regimen was appropriately matched to the new chemotherapy agent, trifluridine-tipiracil, to report whether such symptoms of nausea and vomiting are kept at bay, and to identify a potentially vulnerable interval - immediately after drug approval - when cancer patients may be at risk for CINV because of poor adherence to antiemetic guideline prescribing practices by health care providers.

\section{Methods}

\section{Overview}

The Mayo Clinic Institutional Review Board approved this study. We obtained the identifying information of all patients treated with trifluridine-tipiracil at our institution from the Mayo Clinic Specialty Pharmacy, which uses an electronic prescribing system that contributed to the comprehensiveness of the data set. Patients included those who had participated in a colorectal cancer compassionate-use program before the September 2015 approval of the drug and those who received the drug shortly after its approval. In essence, this retrospective, single-institution study included every patient who received trifluridine-tipiracil for metastatic colorectal cancer in 2015 (January through December); this approach enabled us to systematically report on early firstcycle prescribing practices 9 months before and 3 months after the drug's approval in September of 2015.

\section{Determination of guideline adherence}

This project relied on the National Comprehensive Cancer Network (NCCN) Guidelines (v1.2015, behind paywall) because they had been updated in 2015 (and hence coincided with this project's study dates) to incorporate recommendations specific to oral chemotherapy and because they seemed concordant with other guidelines. ${ }^{10,11}$

Antiemetic prophylaxis for a specific patient was deemed guideline adherent if a version of the recommended NCCN antiemetic regimen had been prescribed during the first cycle of chemotherapy. This regimen consisted of metoclopramide, prochlorperazine, haloperidol, or a 5-hydroxytryptamine receptor antagonist. In contrast, if a patient had been prescribed a more aggressive or less aggressive regimen, such prescribing practices were deemed nonguideline adherent/aggressive (received more prophylaxis than called for) or non-guideline adherent/less aggressive (including no antiemetics), respectively. Again, medical record prescribing determined adherence.

\section{Data reporting}

The primary goal of this study was to report the percentage of patients who had been prescribed a first-cycle antiemetic prophylaxis regimen concordant with NCCN guidelines. Secondary goals included reporting the incidence of nausea and vomiting, the use of rescue antiemetics other than those prescribed up front, the need for an unplanned medical encounter to address nausea and vomiting, and change in antiemetic prescribing before the second chemotherapy cycle. Confidence intervals were calculated with JMP ${ }^{\circledR}$ Pro 10.0.0. This study was too limited in sample size to assess sex-based differences in outcomes.

\section{Results}

\section{Demographics}

This report focuses on 44 patients who received first-cycle trifluridine-tipiracil during the first calendar year of the drug's FDA approval. All patients had metastatic colorectal cancer and had previous exposures to other chemotherapy agents (Table 1). Of note, 28 patients (64\%) had experienced CINV before starting trifluridine-tipiracil and all these patients had been heavily pretreated with multiple lines of chemotherapy.

\section{Guideline adherence}

Patients were most commonly prescribed prochlorperazine and ondansetron prophylaxis for CINV before the first chemotherapy cycle of trifluridine-tipiracil (Table 2): 15 patients were prescribed combination antiemetic ther- 
apy, typically two of the most commonly prescribed single agents with different mechanisms of action. Twenty-five patients (57\%; 95\% confidence interval (CI): 42\%, 70\%) were prescribed antiemetics in a manner consistent with guidelines; 15 (34\%; 95\% CI: 22\%, 49\%) were prescribed antiemetics in a non-guideline-adherent/more aggressive manner (received more prophylaxis than called for); and 4 (9\%; 95\% CI: 4\%, 21\%) were prescribed them in a nonguideline-adherent/less aggressive manner.

\section{Clinical outcomes based on guideline adherence}

In guideline-adherent patients, first-cycle nausea and vomiting occurred in 13 patients (52\%) and 6 patients (24\%), respectively, with 1 patient requiring an unscheduled clinic visit and another an emergency department visit and hospital admission - all for nausea and vomiting (Table 3). In non-guideline-adherent/more aggressive patients, those symptoms occurred in 5 patients (33\%, nausea) and 4 patients (27\%, vomiting), with 1 patient requiring a clinic visit and emergency department visit and another an emergency department visit - again, all for nausea and vomiting. In non-guideline-adherent/less aggressive patients, no nausea or vomiting was reported.

\section{Discussion}

This study examined adherence to antiemetic guidelines in the setting of a soon-to-be-approved or newly approved antineoplastic agent. As hypothesized, a substantial pro-

TABLE 1 Patient characteristics $(\mathrm{N}=44)$

\begin{tabular}{|cc|}
$\begin{array}{l}\text { Characteristic } \\
\text { Mean age at Trifluridine/tipiracil } \\
\text { initiation: } 60 \text { y }(S D, 12)\end{array}$ & $\begin{array}{c}\text { No. of } \\
\text { patients (\%) }\end{array}$ \\
\hline Sex & - \\
\hline Men & $23(48)$ \\
Women & $21(52)$ \\
\hline Trifluridine-tipiracil dose & $43(98)$ \\
\hline 35 mg/m² & $1(2)$ \\
Other & \\
\hline Concurrent bevacizumab? & $43(98)$ \\
No & $1(2)$ \\
Yes & \\
\hline History of CINV? & $5(11)$ \\
No & $28(64)$ \\
Yes & $11(25)$ \\
Unable to determine &
\end{tabular}

CINV, chemotherapy-induced nausea and vomiting portion of patients ( $43 \%$ in this study) were prescribed antiemetics in a nonadherent manner with respect to guidelines, thus identifying the period shortly before and after FDA approval as a particularly vulnerable interval with respect to antiemetic guideline adherence. It is possible that our institution's practice of testing novel chemotherapy agents for the treatment of colorectal cancer prompted a heightened awareness of potential adverse events, leading to greater guideline adherence than might have occurred in other settings and resulting in judicious straying from guideline adherence only when appropriate. ${ }^{12-14}$ Thus, these high rates of poor adherence may in fact represent an underestimate of what one might see in other clinical practices; and, similarly, these rates of symptom control might also be more favorable than those one might see in other clinical practices. To our knowledge, antiemetic prescribing practices with newer chemotherapy agents have not been explored before now, and our data underscore a clear need to do so - particularly during this limited interval when health care providers begin to prescribe new chemotherapy agents for the first time.

It is worth noting that despite the high rates of guideline nonadherence, rates of nausea and vomiting seemed to be comparable in patients prescribed antiemetics in a guideline-adherent manner and those prescribed antiemetics in a non-guideline-adherent/aggressive manner.A small number of patients in both the guideline-adherent and non-guideline-adherent/aggressive groups required rescue medications, unscheduled medical visits for nausea and vomiting, and additional antiemetics during the second cycle of chemotherapy. Of note,none of those interventions occurred in patients who were prescribed antiemetics in a non-guideline-adherent/less aggressive manner. These findings might reflect the fact that the patients had proven themselves to be at risk for nausea and vomiting with previous chemotherapy. Before they became candidates for trifluridine-tipiracil, patients had been heavily pretreated

TABLE 2 Prescribed first-cycle antiemetics ${ }^{a}$
Drug n (\%)

Ondansetron

$16(36)$

Granisetron

1 (2)

Dexamethasone

$1(2)$

Metoclopramide

$1(2)$

Prochlorperazine 34 (77)

Promethazine 3 (7)

Lorazepam $10(23)$

Olanzapine

$1(2)$

a 15 patients were prescribed a combination of these listed agents, with the combination often including 2 agents with different mechanisms of action. 
TABLE 3 Outcomes based on first-cycle guideline adherence

\begin{tabular}{|c|c|c|c|c|}
\hline & $\begin{array}{c}\text { Total, n (\%) } \\
{[N=44]}\end{array}$ & $\begin{array}{c}\text { Guideline-adherent, } \\
\text { n (\%) [n = 25] }\end{array}$ & $\begin{array}{c}\text { Non-guideline- } \\
\text { adherent/ } \\
\text { aggressive, } \\
\text { n (\%) [n = 15] }\end{array}$ & $\begin{array}{c}\text { Non-guideline } \\
\text { adherent/ } \\
\text { less } \\
\text { aggressive, } \\
n(\%)[n=4]\end{array}$ \\
\hline Nausea & $18(41)$ & $13(52)$ & $5(33)$ & $0(0)$ \\
\hline Vomiting & $10(23)$ & $6(24)$ & $4(27)$ & $0(0)$ \\
\hline Rescue antiemetics & $2(5)$ & $1(4)$ & $1(7)$ & $0(0)$ \\
\hline Unscheduled clinic visit* & $2(4.5)$ & $1(4)$ & $1(7)$ & $0(0)$ \\
\hline ED visit* & $3(7)$ & $1(4)$ & $2(13)$ & $0(0)$ \\
\hline Hospital admission* & $1(2)$ & $1(4)$ & $0(0)$ & $0(0)$ \\
\hline Additional antiemetics for $\mathrm{C} 2$ & $2(5)$ & $1(4)$ & $1(7)$ & $0(0)$ \\
\hline
\end{tabular}

with other chemotherapy agents, most had experienced CINV, and many were therefore highly predisposed to nausea and vomiting. These observations underscore the fact that guidelines - even those that are well accepted and widely used - should be implemented in concert with good clinical judgment. ${ }^{10,11}$

This study has shortcomings, most notably its small sample size. However, had we extended our study beyond 3 months of the FDA approval to include more patients, our findings would have reflected more experienced prescribing practices and we thereby would have deviated from our primary goal of assessing antiemetic prescribing practices with only recently-approved and available chemotherapy agents. In this context, this limited sample size aptly serves a primary role of capturing outcomes within a fleeting but critical interval of new drug availability.

In summary, this study found a notable rate of poor guideline adherence when prescribing antiemetics for trifluridine-tipiracil, a new chemotherapy agent of low emetogenic potential. Although the resultant rates of nausea and vomiting suggest that good clinical judgment might have influenced whether or not guidelines were adhered to, these findings nonetheless underscore the need to assess adherence to antiemetic guidelines when new chemotherapy drugs become available and potentially to put in place institutional infrastructure rapidly to promote improved adherence. Such an assessment should be deliberate, formalized, and prompt within individual oncology clinics and cancer centers after a new cancer drug becomes available. In conjunction with clinical judgment, such measures might lead to improved symptom control.

\section{Acknowledgment}

This paper is based on a poster that was presented at the 2016 Palliative Care in Oncology Symposium, on September 10, 2016: Adherence to antiemetic guidelines with a newly approved chemotherapy agent, trifluridine-tipiracil (TAS-102): a single-institution study. Daniel Childs and Aminah Jatoi, Mayo Clinic, Rochester, MN. http://meetinglibrary.asco. org/record/136444/abstract. J Clin Oncol. 2016;34(suppl 26S):abstract 221.

\section{References}

1. CenterWatch. FDA website. FDA approved drugs for oncology: drugs approved for 2015. https://www.centerwatch.com/drug-information/ fda-approved-drugs/therapeutic-area/12/oncology. Last updated April 2017. Accessed June 4, 2016.

2. Navari RM, Aapro M. Antiemetic prophylaxis for chemotherapyinduced nausea and vomiting. N Engl J Med. 2016;374:1356-1367.

3. Kottschade L, Novotny P, Lyss A, et al. Chemotherapy-induced nausea and vomiting: incidence and characteristics of persistent symptoms and future directions NCCTG N08C3. Support Care Cancer. 2016;24:2661-2667.

4. Grunberg SM, Deuson RR, Mavros P, et al. Incidence of chemotherapy-induced nausea and emesis after modern antiemetics. Cancer. 2004;100:2261-2268.

5. Navari RM. The safety of antiemetic medications for the prevention of chemotherapy-induced nausea and vomiting. Expert Opin Drug Saf. 2016; 15:343-356.

6. Gilmore JW, Peacock NW, Gu A, et al. Antiemetic guideline consistency and incidence of chemotherapy-induced nausea and vomiting in US community oncology practice: INSPIRE study. J Oncol Pract. 2014;10:68-74.

7. Mertens WC, Higby DJ, Brown D, et al. Improving the care of patients with regard to chemotherapy-induced nausea and emesis: the effect of feedback to clinicians on adherence to antiemetic prescribing guidelines. J Clin Oncol. 2003;21:1373-1378.

8. Mayer RJ, Van Cutsem E, Falcone A, et al. Randomized trial of TAS-102 for refractory metastatic colorectal cancer. N Engl J Med. 2015;372:1909-1919.

9. Schwartzberg L, Morrow G, Balu S, et al. Chemotherapy-induced nausea and vomiting and antiemetic prophylaxis with palonosetron versus other $5-\mathrm{HT}_{3}$ receptor antagonists in patients with cancer treated with low emetogenic chemotherapy in a hospital outpatient setting in the United States. Curr Med Res Opin. 2011;27:1613-1622. 


\section{Original Report}

10. National Comprehensive Cancer Network. NCCN Clinical Practice Guidelines on Antiemesis, Version1,2015 [behind paywall]. https:// www.nccn.org. Last update not known. Accessed June 4, 2016.

11. Roila F, Herrstedt J, Aapro M, et al. Guideline update for MASCC and ESMO in the prevention of chemotherapy and radiotherapyinduced nausea and vomiting: results of the Perugia consensus conference. Ann Oncol. 2010;21:v232-v243.

12. Grothey A, Van Cutsem E, Sobrero A, et al. Regorafenib monotherapy for previously treated metastatic colorectal cancer (CORRECT): an international, multicentre, randomized, placebo-controlled, phase
3 study. Lancet. 2013;381:303-312.

13. Alberts SR, Sargent DJ, Nair S, et al. Effect of oxaliplatin, fluorouracil, and leucovorin with or without cetuximab on survival among patients with resected stage III colon cancer: a randomized trial. JAMA. 2012;307:1383-1393.

14. Goldberg RM, Sargent DJ, Morton RF, et al. Randomized controlled trial of reduced-dose bolus fluorouracil plus leucovorin and irinotecan or infused fluorouracil plus leucovorin and oxaliplatin in patients with previously untreated metastatic colorectal cancer: a North American Intergroup Trial. J Clin Oncol. 2006;24:3347-3353. 